\title{
SEXUALLY TRANSMITTED INFECTIONS: BULGARIAN PERSPECTIVE
}

\author{
J. Marinova ${ }^{1^{*}}$, B. Parashkevova ${ }^{1}$, R. Gardeva ${ }^{2}$, E. Atanasov $^{3}$, G. Rosenova-Licheva ${ }^{3}$ \\ ${ }^{1}$ Department of Social Medicine and Health Management, Medical Faculty, Trakia University, \\ Stara Zagora, Bulgaria \\ ${ }^{2}$ Medical Center "Derma Guard - Stara Zagora" Ltd., Stara Zagora, Bulgaria \\ ${ }^{3}$ Student, Medical Faculty, Trakia University, Stara Zagora, Bulgaria
}

\begin{abstract}
Introduction: The effective management of sexually transmitted infections (STIs) consists not only of antimicrobial therapy to receive treatment and reduce infectivity, but also in the overall attention and care for the patient's reproductive and sexual health. In July 2019, the WHO called for a concerted effort to ensure access to the services needed to prevent and treat these diseases to everyone and everywhere.

Objective: to study and present European policies and the Bulgarian present situation as a basis for building effective, evidence-based interventions and services for the STIs spectrum with emphasis on syphilis to support the achievement of the WHO strategic goals.

Material and methods: in this study we applied the documentary method: a review of official strategies, reports, articles on the discussed topic.

Results: a review of European and national policies and practices were presented and discussed with emphasis on syphilis. Social, ethical, economic dimensions of the STIs spectrum management were discussed on a national level in order to support the achievement of WHO strategic goals.

Conclusions: The presented review provides a good opportunity to define the priority issues related to the management of the spectrum of sexually transmitted infections for evidence-based health policy ideas, research, will and decisions in Bulgaria.
\end{abstract}

Key words: syphilis, chlamydia, gonorrhoea, trichomoniasis, HIV, epidemic, management, World Health Organization.

\section{INTRODUCTION}

Sexually transmitted infections (STIs) continue to be a significant public health problem with numerous health, socio-ethical and economic consequences for many countries. Access to STIs diagnosis and treatment is an important public health measure, and the costs for the individuals and the health services system could be significant.

The basis of effective action against STIs is a strong health system that is able to provide reliable, effective and equitable care to people,

\footnotetext{
*Correspondence to: Prof. Juliana Krumova Marinova, MD, PhD, Head of the Department of Social Medicine and Health Management, Medical Faculty, Trakia University - Stara Zagora, 11 “Armeiska” Str. , Stara Zagora - 6000, BULGARIA, e-mail: yuliyana.marinova@trakiauni.bg, https://orcid.org/0000-0001-8178-4369
}

both in the public and private sectors. The distinguishing features of such a system are: effective models for providing services that meet the needs of patients; suitably trained and distributed workforce in sufficient numbers; strong health information system; reliable access to basic medical products and technologies; adequate financing of health services; strong leadership and management (1-3).

World Health Organization (WHO) strongly recommends countries to set up and use national standardized STIs treatment protocols to ensure receiving adequate treatment at all levels of health services. The WHO Advisory Group on Sexually Transmitted Diseases Treatment of has reviewed and updated WHO Recommendations for Treatment of STDAssociated Syndromes. One of the cornerstones is the control of STIs, as: 
- it prevents the development of complications and bad consequences;

- $\quad$ it reduces the spread of these infections in the community;

- it offers a unique opportunity for targeted training on HIV prevention (1-3).

The emergence and spread of human immunodeficiency virus (HIV) infection and acquired immunodeficiency syndrome (AIDS) have had a major impact on the management and control of STIs. There are specific conditions where vulnerability and risk are high and where access to basic services related to sexually transmitted infections can be severely compromised, such as in prisons and detention centers, refugee camps, minority groups, the poor and the homeless. States must ensure that the services provided to these strata are equivalent to those available to others, which means health equality is a cornerstone of national health policies (1-3).

\section{AIM}

The aim of this review is to outline the main dimensions of the management of the spectrum of sexually transmitted infections by studying and presenting European policies and the Bulgarian present situation with emphasis on syphilis as a basis for further building effective, evidence-based interventions and services for the STIs spectrum.

\section{MATERIAL AND METHODS}

In this study we applied documentary method on the discussed topic. We reviewed official strategic documents, reports, recommendations, articles of the United Nations, World Health Organization; European Center for Disease Prevention and Control; European Observatory on Health Systems and Policies; National Statistical Institute; National Center of Public Health and Analysis at the Ministry of Health, Bulgarian acts and regulations.

\section{RESULTS AND DISCUSSION}

The effective management of sexually transmitted infections (STIs) consists not only of antimicrobial therapy to treat and reduce infectivity, but also of overall attention and care for the patient's reproductive and sexual health. "More than 1 million sexually transmitted infections (STIs) are acquired every day worldwide": is on the top position of the STIs key facts list (4). The WHO called for a concerted effort to ensure access to the services needed to prevent and treat these
MARINOVA J., et al.

exhausting diseases to everyone and everywhere. The WHO has also published Recommendations for STIs management in the broader context of programs for control, prevention and care. On 14 June 2019 WHO published STIs Key facts, outlined Scope of the problem, the prevention, diagnosis, treatment, STIs case management and concluded that: "Current efforts to contain the spread of STIs are not sufficient" and also "Health services for screening and treatment of STIs remain week" (4).

"Each year, there are an estimated 376 million new infections with 1 of 4 STIs: chlamydia, gonorrhoea, syphilis and trichomoniasis" (4). This spectrum of STIs has a profound impact on the health of adults and children around the world. If left untreated, they can lead to serious and chronic health problems. These include neurological and cardiovascular disease, infertility, ectopic pregnancy, stillbirth and an increased risk of HIV. They are also associated with significant levels of stigma and domestic violence.

"988 000 pregnant women were infected with syphilis in 2016, resulting in over 350000 adverse birth outcomes including $200 \quad 000$ stillbirths and new born deaths". On average, approximately 1 in 25 people worldwide have at least one of these STIs, according to the latest data, and some also have several of these infections at the same time (4).

The Global Strategy for STIs 2016-2021 was adopted by the World Health Assembly in May 2016. The document proposes a rapid expansion of evidence-based interventions and services to address STIs as a public health issue by 2030. People should receive services from who need prevention and treatment of STIs without experiencing financial difficulties (3).

The central to the United Nations Sustainable Development Program Agenda for Sustainable Development "Transforming Our World: The 2030 Agenda for Sustainable Development" is about eradicating poverty and reducing inequality (5). Globally, 150 million people experience a financial catastrophe, and 100 million suffer from impoverishment each year as a result of out-of-pocket health care costs. Therefore, financial security and health equity is fundamental to the program. Countries face the challenge of investing in an expanded program to achieve STIs management goals by 
2030 and beyond, while ensuring long-term sustainability of funding. The trend of increasing domestic funding for HIV and STIs programs is present in some lower income countries $(2,3)$.

Funding for a sustainable response in STIs governance requires an approach that must be embedded in an overall national health strategy in three areas:

- raising sufficient funds to pay for sexually transmitted infections programs, including through public and private domestic funding and external sources;

- creation of fair mechanisms for pooling funds for protection of financial risk;

- optimizing resource use by reducing costs and improving efficiency.

Funding for a sustainable response calls for action in three areas:

- Increasing revenue through innovative financing and new approaches to financing;

- Protection of financial risks and impoverishment;

- Reduce costs and improve efficiency (2, $3)$.

The European Center for Disease Prevention and Control (ECDC) - Stockholm, point out more than 260000 registered cases of STIs, reported by $30 \mathrm{EU}$ countries for the period 2007-2018 (6). About Syphilis: "Since 2010, syphilis notification rates in the EU/EAA have been on the increase, but in recent years this trend seems to accelerate, predominantly among men having sex with men. Similar trends have been observed in high-income countries outside the EU/EAA. While the overall trend remained relatively stable, outbreaks or clusters of syphilis cases have also been reported among heterosexual populations in the EU/EEA. In several highincome countries (e.g. USA, Japan), increases in congenital syphilis occurred in connection with increases in syphilis notifications among women." (6).

In 2010 the incidence of syphilis was 51.6 per 100,000, while in 2011 it increased of 63.3, which are epidemic values. The percentage of notification of syphilis in the EU from 2010 also shows an accelerated trend. Outbreaks or groups of syphilis have been reported among heterosexual and homosexual populations in high-income countries (USA, Australia, Japan, etc.). There has been an increase in syphilis cases in HIV patients (syndemia). If left untreated, syphilis infection can cause serious health damage and facilitate the transmission of HIV infection. The cause of syphilis has its own cycle and now the incidence is reaching its peak. Untreated syphilis infection during pregnancy can seriously damage the pregnancy (loss of the fetus, stillbirth) and lead to congenital syphilis (congenital syphilis) in the newborn (6).

In September 2018 The ECDPC STIs Coordinating Committee raised concerns about an increase in syphilis in the EU and asked ECDPC to prepare an update on the epidemiology of syphilis, assess the current level of risk and indicate response options. When performed a non-systematic literature review of several databases (PubMed, Embase, Scopus) for the period 2007-2018 in order to identify trends, to describe recent outbreaks and to better understand the causes of the growing syphilis epidemic. The characteristics of the cases in the EU and other high-income countries are described - Australia, Canada, Japan, New Zealand, USA. This was combined with an analysis of EU surveillance data 20072017 for syphilis and congenital syphilis and a survey of EU Member States for 2019 on recent trends and changes in surveillance. A total of 189 studies identified by unsystematic syphilis testing and congenital syphilis epidemiology and 78 studies reporting interventional responses with documented effects were used to inform this risk assessment. Responses to the ECDC survey were received from 28/31 Member States. This study found that over the last 10 years, there has been an increased trend of syphilis in the EU and several other high-income countries. Men having sex with men (MSM) are the most affected population in the EU, but in a number of countries the percentage of the affected heterosexual population is increasing. An increase in the diagnosis of syphilis among reported pregnant women leads to an increase in congenital syphilis infections. In the last 10 years, several outbreaks of syphilis $(\mathrm{n}=25)$ and groups of cases $(n=4)$ have been reported in high-income countries - with a range between 5 and more than 1000 cases (6).

\section{Bulgaria}

For Bulgaria, resent official data on syphilis prevalence and on syphilis incidence rates presented the lower level of the indicators 2018 compared to 2017data: 
- Syphilis prevalence rate 2018: 32.1 per 100000 population; 2017: 34.9 per 100 000 population;

- Syphilis incidence rate 2018: 6.4 per 100 000 population; 2017: 7.5 per 100000 population.

- Presented by sex indicators levels are higher for men (7).

Regarding the normative regulation, the Ordinance № 3 of 26 May 2016 is in force on the terms and conditions for conducting diagnostics, prevention and control of syphilis, gonorrhea and urogenital chlamydial infection (8). An Expert Council on HIV and STIs has been established at the Ministry of Health with functions to develop and propose to the Council of Ministers a national policy for prevention and reduction of the spread of AIDS, tuberculosis and STIs. "The National Program for Prevention and Control of HIV and Sexually Transmitted Infections in the Republic of Bulgaria 2017 - 2020 will build on the results achieved in the implementation of the last two national programs in the period 2001-2007 and 2008- 2015, which were supported through the implementation of the Program "Prevention and Control of HIV/AIDS ", funded by the Global Fund to Fight AIDS, Tuberculosis and Malaria in the period 2004-2014" is from the preamble of the currently applied program (9). Priority area 3 of this Program is: "Building a network of services for research sexually transmitted infections". It is underlined that: "Bulgaria faces serious challenges in terms of diagnostics, treatment, registration and notification of STIs. The treatment of STIs is not covered by The National Health Insurance Fund. Not all STIs are confirmed, registered and notified" (9).

"There is a peak in the incidence of STIs in Bulgaria. The poor, the homeless, prostitutes, Roma are among the main and most vulnerable groups - without health insurance, respectively without the opportunity to be treated in hospitals and without financial opportunities to purchase of drugs" (10). The STIs cases follow-up system through the former dermatological and venereological dispensaries has been destroyed and nobody is committed with the STIs cases follow-up and search for sick and contact persons (10). In 2019, only in one of the specialized centers the newly registered cases of syphilis were 30 cases, of which 4 cases of congenital syphilis: Medical
Center "Derma Guard - Stara Zagora" Ltd., Official Report (11). Dermatovenereologists warn that often patients have to pay for their own treatment, and in the absence of financial means, the treatment is at the expense of the respective medical-treatment facility and possibly non-governmental organizations (NGOs) (11).

The problems in Bulgaria concerning the prevention, treatment, medical examination, STIs cases notification could be discussed in the context of: neglect of the Centers for skin and venereal diseases; pre- and post-test counselling; difficulties in establishing and monitoring the epidemiological chain; reliability of statistical data concerning syphilis cases notification and registration. It is also necessary for the treatment to be as individualized as possible (10).

"STIs prevention and control has widespread public health benefits".(12) Important are social, economic, organizational, ethical challenges of the STIs management in the health-political context of limited access to health services at the level of primary and secondary prevention and growing social inequalities in health. It is fair to discuss effective, easily applicable and accessible good practices for the implemented promotional and screening programs in the Republic of Bulgaria, which will allow for universal coverage of vulnerable groups mentioned above, regardless of their health insurance status, place of residence, literacy, income, ethnicity and other characteristics.

We clearly understand the complexity of the discussion in accordance with the principles of biomedical ethics (respect for autonomy, no maleficence, beneficence, justice) (13) and especially their balance for fair and equal access to health services. In this regard we emphasize as the key for the individuals and the social system multidimensional and complex notions as: "justice", "solidarity", "personal responsibility for health", "public interest", "financing". They suggest a broad discussion of different approaches and argumentation. In this context we pointed out only one example: about the persons' health insurance status and the personal responsibility for health and the fact that: "One in every seven Bulgarians lacks health insurance coverage" (14) in the conditions of compulsory health insurance system according to the 
Article 5 of the Health Insurance Act in the Republic of Bulgaria, more than 20 years by now (15). And in the Bulgarian Health Act, the legislator has provided for the persons without health insurance coverage: "Article 82a. "With funds from their own revenues, municipalities can support activities for prevention and treatment of socially disadvantaged, unemployed and other persons who are registered as a permanent address in the municipality." (Effective from 01.01.2019) (16). Above-mentioned status and trends of STIs indicate the need to rethink the mechanisms for financing the activities for prevention, diagnosis, treatment, monitoring of STIs in medical-treatment facilities. We consider it reasonable to support the hypothesis that it is necessary to guarantee funding from the State Budget and the Ministry of Health through the Municipalities, understandably, not all Municipalities have sufficient resources to comply with the provisions of the abovementioned Article 82.a, even in good faith. Such financing system has proven its advantages and benefits with significant social and health dimensions and results for our country during its application until the Medical-Treatment Facilities Act amendment 2010 (17).

\section{CONCLUSIONS}

STIs spectrum management continues to be a major challenge for health systems worldwide. These diseases are a serious threat to public health for the third decade of the 21st century.

A review of European and national policies and practices was presented and discussed with emphasis on syphilis because of the growing syphilis epidemic.

Social, ethical, economic dimensions of the STIs spectrum management were discussed on national level in order to outline the dimensions of the most significant problems of STIs management in Bulgaria.

The presented mini-review provides a good opportunity to define the priority issues related to the management of the spectrum of sexually transmitted infections for better understanding evidence-based health policy ideas, research, decisions and practice in Bulgaria to achieve the WHO strategic goals concerning STIs spectrum.

\section{ACKNOWLEDGEMENTS}

This research was funded by the Medical Faculty, Trakia University - Stara Zagora,
Scientific Project № 12, 2020 in accordance with the Contract for financing a scientific project with the topic: "Socio-ethical and Economic Dimensions of Sexually Transmitted Infections Spectrum Management"

\section{REFERENCES}

1. WHO, Global strategy for the prevention and control of sexually transmitted infections: 2006 - 2015 Breaking the chain of transmission, available from: https://www.who.int/reproductivehealth/pu blications/rtis/9789241563475/en/

2. WHO, Draft global health sector strategies, Sexually transmitted infections, 2016-2021 Report by the Secretariat Sixty-ninth World Health Assembly provisional agenda item 15.1. Document A69/ 33. available from: https://apps.who.int/gb/ebwha/pdf_files/W HA69/A69_33-en.pdf

3. WHO, Global health sector strategy on Sexually Transmitted Infections, 2016-2021 available from: https://www.who.int/publications/i/item/W HO-RHR-16.09

4. WHO, Sexually Transmitted Infections, available from: https://www.who.int/newsroom/fact-sheets/detail/sexuallytransmitted-infections-(stis)

5. Transforming our World: The 2030 Agenda for Sustainable Development , United Nations, 2015, available from: https://sustainabledevelopment.un.org/post2 015/transformingourworld/publication

6. European Centre for Disease Prevention and Control. Syphilis and congenital syphilis in Europe - A review of epidemiological trends (2007 - 2018) and options for response. Stockholm: ECDC; 2019, available from: https://www.ecdc.europa.eu/en/publications -data/syphilis-and-congenital-syphiliseurope-review-epidemiological-trends2007-2018

7. Health Services 2019, National Statistical Institute of the Republic of Bulgaria and the National Center of Public Health and Analyses at the Ministry of Health, (in Bulgarian) available from: https://www.nsi.bg/sites/default/files/files/p ublications/Zdraveopazvane_2019.pdf

8. Ordinance № 3 Of May 26, 2016 On The Order And Conditions For Conducting Diagnosis, Prevention And Control of Syphilis, Gonorrhoea and Urogenital Chlamidia Infection, Issued by the Minister 
of Health, Promulgated State Gazette No. 43 June 7, 2016. (in Bulgarian)

9. National Program for Prevention and Control of HIV and Sexually Transmitted Infections in the Republic of Bulgaria 2017 - 2020: Ministry of Health, National Center of Public Health and Analyses, (in Bulgarian), available from: https://ncpha.government.bg/

10.Petrova E, "Syphilis - contemporary problems" - presentation at the 8th National Science and practical Conference with international participation - Spring Varna Dermatological Days - 21.04.27.04.2014. (in Bulgarian)

11."Derma Guard - Stara Zagora” Ltd, 2019 Annual Report

12.WHO, Report on global sexually transmitted infection surveillance 2018, available from: https://www.who.int/reproductivehealth/pu blications/stis-surveillance-2018/en/

13.Beauchamp, T. L., J. F. Childress, Principles of Biomedical Ethics, Oxford University Press, 1994, pp. 546; (Edition: 7th, 2012, ISBN-13: 978-0199924585 ISBN-10: 0199924589).

14.OECD/European Observatory on Health Systems and Policies (2019), Bulgaria: Country Health Profile 2019, State of Health in the EU, OECD Publishing, Paris/European Observatory on Health Systems and Policies, Brussels, available from:

https://ec.europa.eu/health/sites/health/files/ state/docs/2019_chp_bulgaria_english.pdf

15. Health Insurance Act Promulgated, State Gazette No. 70/19.06.1998, (in Bulgarian), available from: https://www.lex.bg/laws/ldoc/2134412800

16. Health Act Promulgated, State Gazette No. 70/10.08.2004, (in Bulgarian), available from:

https://www.lex.bg/laws/ldoc\%20/2135489 147

17.Medical-Treatment Facilities Act Promulgated SG No. 62/9.07.1999, (in Bulgarian), available from: https://www.lex.bg/laws/ldoc/2134670848 